\title{
Increase of Transient Stability Level of Gas Turbine Power Plant Using FACTS
}

\author{
Sergey Solodyankin ${ }^{1, *}$, Andrey Pazderin ${ }^{2}$ \\ ${ }^{1}$ Branch of JSC "SO UPS" UDC Ural, Yekaterinburg, Sverdlovsk Region, Russia \\ ${ }^{2}$ Ural Energy Institute of the Ural Federal University named after the first President of Russia B. N. Yeltsin, Yekaterinburg, Sverdlovsk \\ Region, Russia
}

\begin{abstract}
The article is devoted to the development of the mathematical models of modern devices of flexible alternating current transmission systems (FACTS) when calculating the modes and stability of power systems and to the analysis of influence of the specified devices on transient stability of the generators. The considered scheme contains the generators with the gas turbine drive that have electromechanical parameters providing lower level of transient stability compared to units of higher power rating, which in some cases requires implementation of measures for transient stability enhancement. As examples of FACTS the following devices have been considered: compensating device based on voltagesourced converter (STATCOM), static synchronous series compensator (SSSC) and the unified power flow controller (UPFC). The known examples of mathematical models of FACTS devices vary in complexity. For a preliminary assessment of the effectiveness of the FACTS devices, it is proposed to use simplified models that adequately reflect their impact on transients. The use of models made it possible to establish a positive impact of the devices on transient stability of generating equipment in case of short circuits in the electric network. The important conclusion here is that the use of the UPFC device based on two converters (with a corresponding increase in cost) compared to one converter device (STATCOM or SSSC) slightly increases the level of transient stability and the limit time of short circuit disconnection. The proposed method of simulating the FACTS devices is suitable for numerical calculations of transient processes in electric power systems, in particular, to estimate the impact on the transient stability level of the parallel operation of power plants in case of disturbances.
\end{abstract}

\section{Introduction}

The aim of the article is to describe the mathematical modeling technique of the devices of flexible alternating current transmission systems and the analysis of their efficiency in terms of influence on the level of transient stability of parallel operation of the power plant equipped with generators with deteriorated electromechanical parameters. The devices of the regime regulation based on the power electronics allow to solve problems with providing required indicators of stability and reliability of electrical power systems and also decrease total costs of the electrical energy transfer because the new network construction is not necessary [1-6]. These devices are used to increase the indicators of controllability and capability of electricity transmissions and define properties of flexible alternating current transmission systems. Electrotechnical network equipment of the FACTS is capable to solve problems with the operation modes optimization in electrical power systems by several criteria at once: transmission capability, level of technological losses, stability, redistribution of the power flows, quality of the electric energy, etc. [7-8]. Besides, implementation of the FACTS allows to reduce or eliminate the action adjustments from emergency control systems in a number of circuit-mode situations.

Due to the development of the FACTS technologies, it is relevant to develop the methods of assessing the effectiveness of such devices for further promotion in the United Power System of Russia.

\section{Calculation scheme}

As a design scheme, in which a gas turbine power plant of relatively low capacity is connected by a power transmission line to a powerful load, the voltage at the connection point can be considered constant (Figure 1). A compensating device of parallel/series action, socalled unified power flow controller is in the middle of the line. The device consists of two voltage-sourced converters connected by a direct current link through which the converters can exchange active power; each of the converters generates independently reactive power.

Such scheme is rather widespread in electrical supply systems of oil and gas extraction complexes. The output of the power plant is $100 \mathrm{MW}$ (4 machines on $25 \mathrm{MW}$, $\cos \varphi=0.9$ ). Electromechanical parameters of generators:

\footnotetext{
Corresponding author: solodsal@mail.ru
} 
the synchronous, transient and subtransient reactances are respectively equal to $\mathrm{xd}=1.98$ p.u., $\mathrm{x}^{\prime} \mathrm{d}=0.3$ p.u., $\mathrm{x} " \mathrm{~d}=0.216$ p.u. The Value of the mechanical inertial constant of the unit: $\mathrm{Tj}=2.3 \mathrm{~s}$. These circumstances cause reduced level of the transient stability in short circuits. The connection with the receiving end of the power system is performed by double-circuit power transmission line of nominal voltage $110 \mathrm{kV}$ with length of $60 \mathrm{~km}$ (made by steel-aluminum wire $240 \mathrm{~mm}^{2}$ ). Different types of multi-phase short circuits (SC) at the beginning of the transmission line (the bus with $\mathrm{U} 1$ ) are used as disturbances [9]. The aim of this article was the analysis of the FACTS devices influence on the electromechanical transition processes at disturbance and definition of limit time of fault clearing at different structure of the compensating device.

It was assumed that the power of each of the UPFC converters was 0.225 p.u. (25 MVA), and the power S of the power plant was taken as the basis power.

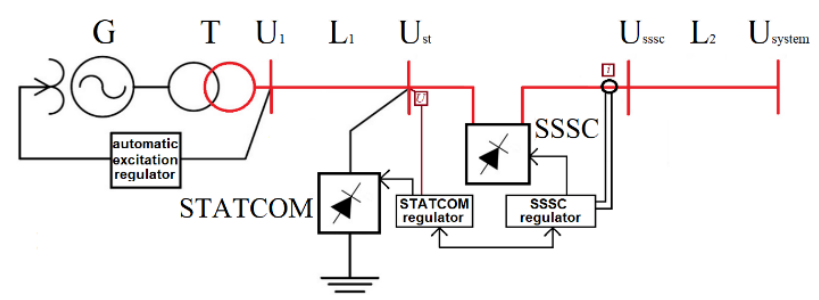

Fig. 1 Design scheme with FACTS device.

\section{Modeling of FACTS devices}

\subsection{Mathematical model of STATCOM}

The following dynamic model as a system of algebraic differential equations is introduces in order to carry out calculations of electromechanical transient processes of electric power system with a device of STATCOM type.

The input variable is the voltage on the high voltage buses of network at the connection point with $U_{\text {st }}$ set by the projections of $U_{\mathrm{q}}$ and $U_{\mathrm{d}}$ on the coordinate axes of $\mathrm{q}$ and $\mathrm{d}$ combined with a rotor of the equivalent generator.

The output power of STATCOM $S_{\text {st }}$ is set by in basic data.

The increment of the electromotive force (EMF) STATCOM $\Delta E_{\mathrm{st}}$ is calculated on the basis of the following transfer function:

$$
\Delta E_{s t}=\frac{K_{s t} \cdot\left(U_{s t}-U_{s e t}\right)}{1+p T_{v}}
$$

where $U_{\text {set }}$ is the set value of voltage setpoint;

$K_{\text {st }}$ is the coefficient of regulation of the voltage deviation channel $\left(K_{\mathrm{st}}=-5 \ldots-100\right.$ units of EMF STATCOM/unit of voltage);

$T_{\mathrm{v}}$ is the time constant of voltage deviation channel $\left(T_{\mathrm{v}}=0.02 \mathrm{~s}\right)$.

Since the simulation uses a common coordinate system q, d associated with the mathematical model of the generator, the coefficients of the EMF increment distribution along the $\mathrm{q}$ and $\mathrm{d}$ axes are calculated:

$$
\begin{aligned}
& K_{q}=a b s\left(U_{q}\right) / U_{s t} \\
& K_{d}=a b s\left(U_{d}\right) / U_{s t}
\end{aligned}
$$

Components of STATCOM currents along the axes $q$ and $d$ are calculated in accordance with relations linking components of its EMF and voltages at its connection point:

$$
I_{q s t}+j I_{d s t}=E_{q s t}+j E_{d s t}-U_{q}-j U_{d} \cdot j b_{s t}
$$

it follows that

$$
\begin{gathered}
I_{q s t}=-b_{s t} \cdot E_{d s t}-U_{d} \\
I_{d s t}=b_{s t} \cdot E_{q s t}-U_{q}
\end{gathered}
$$

Expressions in parentheses represent projections of increments of EMF STATCOM respectively on $\mathrm{d}$ and $\mathrm{q}$ coordinate axes, so q, d - components of currents are calculated from expressions:

$$
\begin{gathered}
I_{q s t}=-b_{s t} \cdot \Delta E_{s t} \cdot K_{d} \cdot \operatorname{sign} U_{d} \\
I_{d s t}=b_{s t} \cdot \Delta E_{s t} \cdot K_{q} \cdot \operatorname{sign} U_{q}
\end{gathered}
$$

The value of the EMF increment is limited in such a way that the module of current STATCOM equal to the $\Delta E_{s t} \cdot b_{s t}$ cannot exceed the $S_{\mathrm{st}}$ device power size set in relative units.

\subsection{Mathematical model of SSSC}

The mathematical model similar to model STATCOM is developed for the SSSC. It is supposed that the device can independently operate as the static synchronous series compensator and also as a part of the unified power flow controller (UPFC) that includes STATCOM exchanging active power with SSSC (according to regulation parameters of the last). Power links of two devices are presented in the set of constraints.

Input variable is the value of current $I_{m}$, proceeding on the transformer which is switched on in series in a transmission line in device connection point, set by the projections $I_{q}$ и $I_{d}$ on coordinate axes of the general coordinate system $\mathrm{q}$ and $\mathrm{d}$.

Inductive reactance of SSSC transformer is taken in relative units equal to $\mathrm{x}_{\mathrm{sssc}}=0.02 \ldots 0.04 \mathrm{p}$.u.

The power of the SSSC $S_{\text {ssse }}$ is set in the basic data (usually taken equal to the power rating of the STATCOM).

The EMF control signal of SSSC is calculated from the known current deviation using the following transfer function:

$$
\Delta I_{m}=\frac{K_{0 i}\left(I_{m}-I_{s e t}\right)}{1+p T_{i}}
$$

where $I_{\text {set }}$ is a setting value on current;

$K_{0 \mathrm{i}}$ is a coefficient of regulation of the current deviation channel $\left(K_{0 \mathrm{i}}=10 \ldots 100\right.$ units of EMF $\mathrm{SSSC} /$ unit of current); 
$T_{i}$ is a time constant of the current deviation channel $\left(T_{i}=0.01 \mathrm{~s}\right)$.

The projections of SSSC EMFs on axes $\mathrm{q}$ and $\mathrm{d}$ are calculated with transfer functions using additional signal of generator rotor speed deviation $s$ from the synchronous speed (time constants $T_{\text {ssse }}$ и $T_{0 \omega}$ are $0.01 \mathrm{~s}$ ):

$$
\begin{aligned}
\Delta E_{q s s c} & =\frac{\Delta I_{m} \cdot x_{s s s c} \cdot I_{\text {dreg }}}{1+p T_{\text {sssc }}}+\frac{k_{0 \omega} \cdot s}{1+p T_{0 \omega}} \\
\Delta E_{\text {dsssc }}= & \frac{-\Delta I_{m} \cdot x_{s s s c} \cdot I_{\text {qreg }}}{1+p T_{\text {sssc }}}+\frac{k_{0 \omega} \cdot s}{1+p T_{0 \omega}}
\end{aligned}
$$

Where $I_{\text {dreg }}, I_{\text {qreg }}$ are the components of SSSC current, determined in accordance with the control angle $\rho$ of (when SSSC is a part of UPFC, the angle $\rho$ is generally different from zero):

$$
\begin{aligned}
& I_{\text {dreg }}=I_{q} \cdot \sin \rho+I_{d} \cdot \cos \rho \\
& I_{\text {qreg }}=I_{q} \cdot \cos \rho-I_{d} \cdot \sin \rho
\end{aligned}
$$

When SSSC operates as a part of UPFC, active power exchange with the network depends on STATCOM capabilities in accordance with limitations determined by the power rating of the devices. Active current components $I_{\text {qsssc }}, I_{d s s s c}$ of SSSC (needs for calculating the power exchange between voltage-source converters) are calculated according to the value of the measured active power of SSSC $P_{S S S c_{1}}$. Formally equating the value of measured reactive power of SSSC $Q_{s s s c_{1}}$ to zero we can obtain the following relations that determine $\mathrm{d}, \mathrm{q}$ - components of the SSSC current corresponding to the active power:

$$
\begin{gathered}
I_{q s s s c}=\frac{P_{s s s c_{1}}}{\frac{\Delta U_{d s s s c}{ }^{2}}{\Delta U_{q s s s c}}+\Delta U_{q s s s c}} \\
I_{d s s s c}=I_{q s s s c} \cdot \frac{\Delta U_{d s s s c}}{\Delta U_{q s s s c}}
\end{gathered}
$$

Due to the active power exchange of STATCOM with the electrical power network and through the DC link with SSSC, additional components are entered into equations (6) and (7):

$$
\begin{gathered}
I_{q s t}=-b_{s t} \cdot \Delta E_{s t} \cdot K_{d} \cdot \operatorname{sign} U_{d}+I_{q s s s}, \\
I_{d s t}=b_{s t} \cdot \Delta E_{s t} \cdot K_{q} \cdot \operatorname{sign} U_{q}+I_{d s s s c}
\end{gathered}
$$

The additional voltage increments of SSSC are calculated from obtained components of EMF (formulas (9), (10)) taking into account limitations determined by the active power exchange with STATCOM in accordance with its own reactive power loading. Voltage increments introduced by the SSSC are limited so that they do not exceed preset values of the converter power rating.

The restriction system is constructed so that SSSC has priority over STATCOM as active power supply with SSSC reduces reactive power generation of STATCOM, using for the voltage regulation in connection point.
The simulation results, shown in the next section of this article, had been obtained using the Dymola software. The correctness of the devices simulation performed within the framework of the article was confirmed by comparing transients in normative disturbances with processes calculated in Matlab/Simulink (SimPowerSystems Blockset).

\section{Obtained influence transient processes results and limit time of fault clearing}

The fault clearing limit time was determined in case of double-phase-to-ground short circuit (SC) and threephase $\mathrm{SC}$ at the beginning of the power transmission line.

The impact of single operating mode of devices (STATCOM/SSSC) and the total composition of UPFC was assessed.

In all cases it was assumed that the generator is equipped with a fast-excitation control system (digital model automatic excitation regulator of strong action based on semiconductors was used), which provided double excitation forcing at the initial stage of the process and subsequent continuous adjustment of voltage regulation, which caused damping of rotor oscillations. The limit times results of SC clearing calculation are summarized in Tables 1 and 2.

The cut-off time limit was determined at the first angle fluctuation.

Table 1. Limit value of time of double-phase-to-ground SC disconnection.

\begin{tabular}{|c|c|}
\hline Calculation option & Time SC, $\mathbf{s}$ \\
\hline Without additional devices & 0.1592 \\
\hline STATCOM & 0.1635 \\
\hline SSSC & 0.1667 \\
\hline UPFC & 0.1710 \\
\hline
\end{tabular}

Table 2. Limit value of time of three-phase SC disconnection.

\begin{tabular}{|c|c|}
\hline Calculation option & Time SC, $\mathbf{s}$ \\
\hline Without additional devices & 0,1110 \\
\hline STATCOM & 0,1130 \\
\hline SSSC & 0,1138 \\
\hline UPFC & 0,1155 \\
\hline
\end{tabular}

When analyzing the results given in Tables 1 and 2, it is necessary to keep in mind that the increment of rotor angle value in the short circuit has a quadratic dependence on the disturbance duration. The data of Tables 1 and 2 show that in order to ensure stability with considered disturbances, it is reasonable to use relay protections and switching devices, which ensure the time of SC clearing at the level of $0.1 \mathrm{~s}$.

In determining the limit times of the SC disconnection, rather small values of the control coefficients of the voltage deviation channel of $\operatorname{STATCOM}\left(K_{\mathrm{st}}=-10\right)$ and the current deviation channel of $\operatorname{SSSC}\left(\mathrm{K}_{0 \mathrm{i}}=10\right)$ were used, which provide satisfactory stabilization of the specified values during transient processes. 
Despite the relatively small increase in the SC cut-off time limit, the effect of the FACTS devices on the transition process is positive. To illustrate this conclusion, Figures 2 and 3 compare processes caused by a double-phase-to-ground SC without and with FACTS devices.

Figure 2 compares curves illustrating the change of power transmission angle and electromagnetic power of the generator in case of double-phase-to-ground SC. We can observe significant decrease in the amplitude value of the angle $\delta_{\Sigma}$ in the first fluctuation.

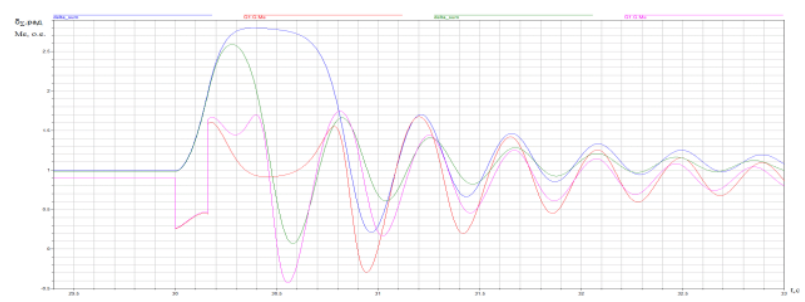

Fig. 2 Change of the angle and electromagnetic power of the generator at double-phase-to-ground SC with duration of short circuit $\Delta \mathrm{t}_{\mathrm{SC}}=0,1592$ (without STATCOM - blue and red curves, with STATCOM green and violet curves respectively)

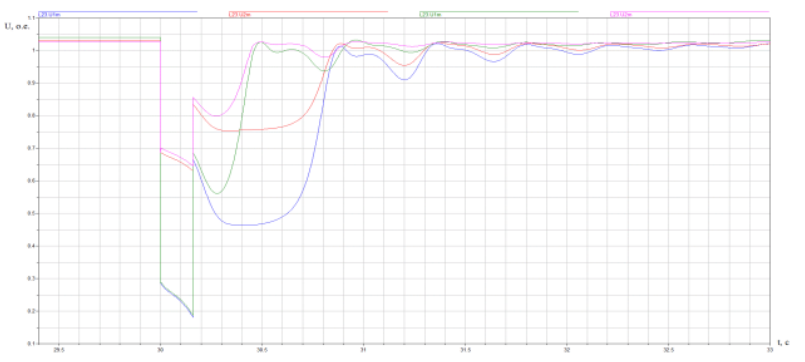

Fig. 3 Change of voltage at the beginning of the line L1 $\left(\mathrm{U}_{1}\right.$ - blue and green curves) and at the installation point of STATCOM ( $\mathrm{U}_{\mathrm{st}}$ - red and purple curves); blue and red curves are when STATCOM is switched off, green and violet curves are when STATCOM is switched on.

An important factor affecting the process is the change of the voltage value at the midpoint of the power transmission line [10-13]. As shown in Figure 3, without STATCOM the process is characterized by a long-term reduction of voltage values $\mathrm{U}_{1}$ and $\mathrm{U}_{\mathrm{st}}$.

It should be noted that the main variables (transmission angle, electromagnetic generator power, $\mathrm{U}_{1}$ и $\mathrm{U}_{\mathrm{st}}$ voltage) change slightly in comparison when SSSC is used and when the full structure of UPFC is switched on. Therefore the specific structure of the device should be considered with technical and economic comparison, considering that UPFC cost (consists of two voltage-source converters) exceeds twice the cost of STATCOM [14]. Obtained considerations are also confirmed by the relatively small dependence of SC disconnection limit times on the structure of the compensating device.

\section{Conclusions}

The method of mathematical modeling of fast-acting devices of the FACTS based on presentation of them by regulated sources of EMF, related by the system of mutual restrictions has been developed. Such device models were verified by comparing transients in disturbances with those calculated in the Matlab/Simulink complex. The presentation of devices in the form of sources of controlled EMF allows to preliminary assess the efficiency of their application in order to increase the level of transient stability.

The influence of the FACTS devices on the limit time of short circuit disconnection has been considered and it has been noted that the use of a device with one converter (STATCOM or SSSC) is more effective. Application of UPFC device based on two converters (with corresponding increase of cost) in comparison with one converter device (STATCOM or SSSC) slightly increases the level of transient stability and the limit time of short circuit disconnection.

\section{References}

1. V.I. Kochkin, Y.G. Shakaryan, Application of the FACTS in power systems (TORUS PRESS, Moscow, 2011)

2. A.V. Pazderin, S.A. Solodyankin, Power plants, 5, pp. $38-41$ (2009)

3. A.V. Pazderin, S.A. Solodyankin, Power plants, 11, pp. $34-39$ (2012)

4. A. S. Lyamov, A.V. Pazderin, S.A. Solodyankin, News of STC UES, 1(76), pp. 77 - 86 (2017)

5. N. G. Hingorani, L. Gyugyi, Understanding FACTS (IEEE Press, N.Y., 2000)

6. M. Eremia, Advanced Solutions in Power Systems (IEEE Press, Hoboken, 2016)

7. K.R. Padiyar, FACTS Controllers in Power Transmission and Distribution (New Age International Publishers, New Delhi, 2007)

8. X.P. Zhang, C. Rehtanz, B.C. Pal, Flexible AC Transmission Systems: Modeling and Control (Springer-Verlag Berlin Heidelberg, 2006)

9. Methodological guidelines on the stability of power systems (Approved by the Ministry of Energy of Russia, 2018)

10. B.N. Abramovich, Y.A. Sychev, Industrial energy, 9, pp. 18-22 (2016)

11. B.N. Abramovich, Y.A. Sychev, Note of Mining institute, 217, pp. 132-139 (2016)

12. B.N. Abramovich, Y.A. Sychev, Mountain information and analytical bulletin (scientific and technical magazine), S7, pp. 737-744 (2015)

13. B.N. Abramovich, Y.A. Sychev, Oil Industry, 9, pp. 120-123 (2016)

14. Ch.Kiran Kumar, M.Sudheer Kumar, V.SriramBabu, S.Nagulmeera, IOSR-JEEE, 4, Issue 6, pp. 53-61 (2013) 